\title{
Multiband modelling of the Sun as a variable star from VIRGO/SoHO data
}

\author{
A. F. Lanza ${ }^{1}$, M. Rodonò ${ }^{2,3}$, and I. Pagano ${ }^{1}$ \\ 1 INAF - Osservatorio Astrofisico di Catania, Via Santa Sofia, 78 Città Universitaria, 95123 Catania, Italy \\ e-mail: nlanza@ct.astro.it \\ 2 Dipartimento di Fisica e Astronomia, Università degli Studi, Via Santa Sofia, 78 Città Universitaria, 95123 Catania, Italy \\ 3 Istituto Nazionale di Astrofisica (INAF), Viale del Parco Mellini 84, 00136 Roma, Italy \\ e-mail: [mrodono;ipagano]@ct.astro.it
}

Received 8 January 2004 / Accepted 4 June 2004

\begin{abstract}
The time series of total solar irradiance (TSI) and optical spectral irradiance at 402, 500 and $862 \mathrm{~nm}$ (SSIs) obtained by the VIRGO experiment on board the satellite SoHO are analysed in order to model their variability in the framework of a purely stellar-like approach. The different time scales of variability are estimated by means of the pooled variance method revealing the growth and decay of sunspots and faculae in active regions, as well as their rotationally modulated visibility. The determination of the rotation period of the Sun from the time modulation of the TSI and SSIs is made difficult by the short lifetimes of photospheric brightness inhomogeneities in comparison to the rotation period. Only during the phases with the lowest level of activity of solar cycle 23, when the variability is dominated by long-lived faculae, is it possible to determine the truly solar synodic period. The simultaneous modelling of the rotational modulation of the TSI and SSIs can be performed by means of a simple stellar-like approach which extends the model we previously applied to the TSI modulation alone (Lanza et al. 2003). Our model yields residuals about 20-30 times smaller than the amplitudes of the TSI and SSI variations in all the phases of the 11-yr activity cycle. The determination of the model parameters, including the temperature of the surface brightness inhomogeneities and the trade-off among them are discussed and compared with the results obtained with different modelling approaches. The advantages and the drawbacks of applying the model to other stars are also considered.
\end{abstract}

Key words. Sun: activity - Sun: rotation - stars: activity - stars: rotation - stars: planetary systems

\section{Introduction}

The variation of the solar irradiance has been monitored by several space-borne instruments since the late seventies (e.g., Chapman 1987; Fröhlich \& Lean 2002). In particular, the VIRGO experiment on board the satellite SoHO has provided us with the most accurate measurements of the disk-integrated flux versus time both in the bolometric and in three optical passbands centered around 402, 500 and $862 \mathrm{~nm}$ (Fröhlich et al. 1995; Fröhlich et al. 1997a,b). The measurements of the bolometric flux and those in the three optical passbands have been converted into Total Solar Irradiance (TSI) and Spectral Solar Irradiance (SSI) values, respectively, that allow us to study the optical variability of the Sun as a star in the course of the 11-yr activity cycle because the available time series span from the beginning of 1996 , close to the cycle 23 minimum, up to the 2000-2001 maximum and then to the presently declining phase.

The variability of the solar irradiance is due to several physical processes which are not yet satisfactorily understood. Apart from the p-mode oscillations, which dominate the variability on time scales of the order of a few minutes, the variations of the spectral as well as total irradiance have been modelled by considering the flux perturbations associated with active regions, which are the manifestations of the surface magnetic fields. Specifically, most of the solar variability on time scales ranging from a few days up to a few hundred days could be accounted for as the effect of the brightness perturbations due to sunspots and faculae (Fligge et al. 1998; Unruh et al. 1999; Fligge et al. 2000; Krivova et al. 2003). The correlation between measured and modelled total irradiance variations was $\sim 95 \%$, whereas the correlations among the observed and modelled SSI variations were somewhat lower, possibly due to systematic errors affecting SSI data and arising from the limited knowledge of the long-term degradation of the optical detectors (Fligge et al. 2000; Krivova et al. 2003). The effect of a background irradiance variation due to an unresolved diffuse network outside active regions or to some unspecified process was invoked to account for the variability on time scales comparable to the 11-yr solar cycle (Foukal \& Lean 1988; Foukal et al. 1991; de Toma et al. 2001), but the recent analysis by Krivova et al. (2003) suggested that the brightness inhomogeneities associated with surface magnetic fields as mapped by MDI may suffice to explain the observed variations. 
A better understanding of the solar variability can be reached by comparing the Sun with other solar-like stars. In the Sun the optical variability induced by the largest active regions is of the order of a few $10^{-3}$ in relative units, whereas in the most active stars it may reach a few tens of percents (Rodonò et al. 1986; Henry et al. 1995). Selection effects favour the study of solar-like magnetic activity in the most active objects, whereas the possibility of studying stars with an activity level comparable to that of the Sun is severely limited because an accuracy of the order of a few millimag could be reached from the ground only for the seasonally averaged magnitudes of some limited samples of late-type stars (cf. Radick et al. 1998). However, the situation will change in the next years thanks to the highly accurate photometry made possible by space-borne dedicated telescopes, such as the COROT and Kepler missions (e.g., Baglin et al. 2001). The main purpose of such instruments is to detect stellar oscillations and to search for Earth-like planets through the periodic light dimming produced by their transits across the disk of the parent star (e.g., Jenkins et al. 1996; Defay et al. 2001; Jenkins 2002).

To assess the information that can be derived by photometric time series with a relative accuracy of the order of $10^{-4}$, the best approach is to test the proposed methods on the VIRGO data because for the Sun time and spatially resolved data are available for performing a detailed comparison.

In the framework of such an approach, we already analysed the TSI time series provided by VIRGO/SoHO by means of a simple model inspired by the methods used to model the rotational modulation of magnetically active stars. In the case of the Sun, it is necessary to take into account the intrinsic evolution of active regions on time scales of the order of a week to fit the irradiance changes properly. Our model proved to be capable of accounting for most of the TSI variability, leaving residuals with a relative amplitude of the order of $(1-2) \times 10^{-4}$, i.e., about 20 times smaller than the TSI variability amplitude during solar maximum (Lanza et al. 2003, hereinafter Paper I).

In the present paper, we extend our approach performing a simultaneous modelling of the total and spectral irradiances at 402,500 and $862 \mathrm{~nm}$ and discuss the advantages and drawbacks of the modelling of multiband data. Unfortunately, the long-term systematic errors affecting the SSI measurements prevented us from studying the long-term effects related to the 11-yr cycle, as in our previous analysis of the TSI time series.

The approach discussed in the present paper may be applied to improve the efficiency of the techniques to detect planetary transits and to provide information on the variability related to surface active region evolution and stellar rotation (cf. Paper I; and Aigrain et al. 2004).

In the following section, we present the VIRGO/SoHO irradiance data and discuss their characteristics relevant for our investigation. The rotational modulation signal is analysed by means of different techniques to assess the possibility of deriving the true solar rotation period for different levels of activity. In Sect. 3, our approach for the multiband modelling of the variability of the Sun as a star is introduced and its main assumptions are discussed in the framework of Paper I. The results of its application to the TSI and SSI time series are presented in Sect. 4 whereas Sect. 5 summarizes the advantages and drawbacks of the proposed method in comparison to similar approaches.

\section{The total solar irradiance and spectral irradiance time series and their variability}

The VIRGO experiment carries two types of radiometers to measure the TSI and the SSIs, respectively. The active cavity absolute radiometers DIARAD and PMO6V provide measurements of the bolometric disk-integrated flux of the Sun from which the TSI are derived. The three sunphotometers (SPM) based on interference filters and silicon detectors provide measurements of the disk-integrated solar flux in three channels of $5 \mathrm{~nm}$ bandwidth centered at 402, 500 and $862 \mathrm{~nm}$, respectively. Actually two identical copies of each instrument are flying on SoHO, one maintained in nearly continuous operation and the other exposed occasionally to evaluate the degradation of the former due to the exposure to solar radiation. Fröhlich et al. (1995) and Fröhlich et al. (1997a) described the basic operation principles and performances of the radiometers and the sunphotometers, as well as the procedures to convert their measurements into physical units and the correction to be applied for known a priori instrument-related effects, e.g., the influence of ambient temperature. The further reduction of the measured flux to $1 \mathrm{AU}$ distance and to zero radial velocity leads to the socalled level 1.0 data, which can be accessed through the Public SoHO Archive. They consist of 1-min averaged measurements for PMO6V and SPM and 3-min averages for DIARAD.

The TSI data analysed in this paper were retrieved from the anonymous ftp site of the Physikalisch-Meteorologisches Observatorium Davos ${ }^{1}$ and are those listed in the file virgo_tsi_h_v5_007_0308.dat that reports the hourly means of the solar total irradiance at level 2.0. Details of the assessment of the long-term behaviour of the radiometers and the correction procedures to obtain level 2.0 TSI data can be found in Anklin et al. (1999), Fröhlich \& Finsterle (2001), Crommelynck et al. (2004) and Fröhlich (2003). The SSI data, corrected for the known a priori effects, but not for the effects of the long-term degradation of the SPM, have been extracted from file spma_level1.5_hourly.dat, which lists the hourly means for the three optical channels. For details of the VIRGO data see the web site of the PhysikalischMeteorologisches Observatorium Davos - World Radiation Center $^{2}$ (Fröhlich \& Wehrli 2003). We made use of the TSI and SSI daily means in the file spma_level2_daily.dat to obtain a series of hourly TSI data with the same long-term detrending of the SSI level 2.0 time series. The time series obtained in this way begin on 7 April 1996 and end on 30 November 2002. They show occasional gaps with typical durations of a few days, except for one gap lasting four months in mid 1998, when spacecraft tracking was lost.

The relative standard deviations of our hourly means were computed from the 1-min measurements in the corresponding

\footnotetext{
1 ftp://ftp.pmodwrc.ch/pub/data/irradiance/virgo - data files that can be freely used provided that the original contribution of the VIRGO team is acknowledged.

2 http://www.pmodwrc.ch/pmod.php?topic=project_space
} 
level 1.0 data retrieved from the Public SoHO Data Archive. They do not show significant changes from the minimum to the maximum of solar cycle 23 and are equal to $2.0 \times 10^{-5}$ for the TSI, $7.2 \times 10^{-5}$ for the $402 \mathrm{~nm}$ channel, $5.0 \times 10^{-5}$ for the $500 \mathrm{~nm}$ channel and $2.9 \times 10^{-5}$ for the $862 \mathrm{~nm}$ channel, respectively. These values give us an estimate of the accuracy of the individual hourly means, but systematic errors may be significantly larger, especially for the SSI values.

The characteristic time scales of the variations that are displayed by a time series can be estimated by means of the socalled pooled variance method (Dobson et al. 1990; Donahue \& Baliunas 1992; Donahue 1993; Donahue et al. 1997a,b). For a given time interval, the data are binned into segments having the length of that interval, and the average variance of the set of these segments, that is the pooled variance, is computed. The pooled variances for the three SSI channels and the TSI are plotted as a function of the length of the time interval $\tau$ in Fig. 1, where the upper limit of $\tau$ was set at 130 days because the SSI time series changes cannot be trusted for longer time scales (cf. Frölich \& Wehrli 2003). As a matter of fact, the TSI variation can be reliably determined over the entire time span of the VIRGO observations and we refer the reader to Paper I for the complete plot of the pooled variance of the TSI and its discussion.

A slope change of the pooled variance at a given time scale indicates that a new process causing an increase of the variance of the data is becoming effective on that time scale. On the other hand, the constancy of the pooled variance on a range of time scales indicates that no additional process inducing variability of the data is becoming effective on that range of time scales. The pooled variance in the optical band increases from longer to shorter wavelengths at a given time interval $\tau$, indicating that the dominant contribution to the solar variability is that of the faculae, whose contrast rises steeply toward the UV (cf. Unruh et al. 1999).

The analysis of SSI time series reveals two slope changes not detected in the pooled variance diagram of the TSI presented in Paper I. The first takes place at $\tau \simeq 9 \mathrm{~d}$ and is apparent in all the three optical bands. It may be related to the typical growth time scale of sunspot groups which produce dips in the irradiance time series. The second slope change occurs at $\approx 60 \mathrm{~d}$ in the 402 and $500 \mathrm{~nm}$ channel variances, but does not appear to be significant in the $862 \mathrm{~nm}$ channel and in the total irradiance. We suggest that this slope change indicates the typical lifetime of the large faculae dominating solar irradiance variations (Lawrence 1987; Foukal et al. 2000). Note that their lifetime is comparable with those estimated by the same technique for a sample of chromospherically active solar-like stars monitored by the Ca II H\&K Mt. Wilson project (Donahue et al. 1997a).

The pooled variance analysis is independent of the shape of the flux time variation. Since we are interested in modelling the rotational modulation, it is useful to apply tools to look for a periodicity of the data in the characteristic period range of solar active region rotation, such as the Morlet wavelet (e.g., Hempelmann \& Donahue 1997; Hempelmann 2002). Moreover, we can apply the Scargle periodogram technique (Scargle 1982; Horne \& Baliunas 1986) and the Phase

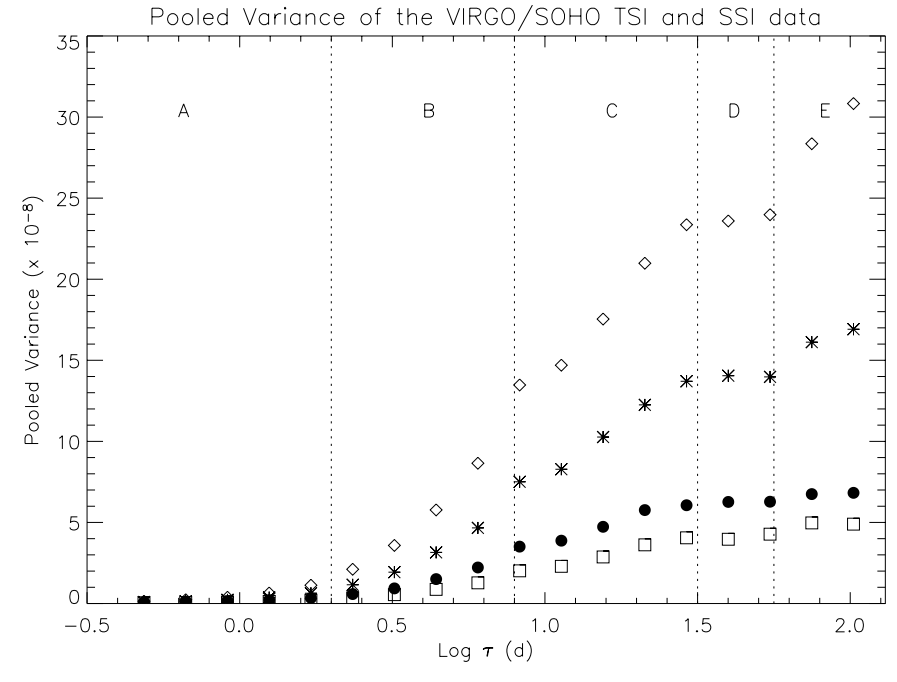

Fig. 1. The pooled variance of the relative variations of the TSI and SSIs as a function of the time interval $\tau$ chosen to bin the data. The different symbols refer to the different spectral passbands as follows: open diamonds $-402 \mathrm{~nm}$; asterisks $-500 \mathrm{~nm}$; open squares $862 \mathrm{~nm}$; filled circles - total solar irradiance. The main ranges of variation of the pooled variance are indicated with A, B, C, D and E and are separated by vertical dotted lines at $2,8,32$ and 56 days, respectively.

Dispersion Minimization method (Stellingwerf 1978) to seasonal subsets of the TSI and SSI of length $158 \mathrm{~d}$ (cf. Paper I).

The Morlet wavelet is capable of retrieving the solar synodic period up to the beginning of 1998, except in the $500 \mathrm{~nm}$ channel, whereas the rotational modulation signal is masked by the active region evolution during the years close to the activity maximum. A splitting of the power into up to four ridges is observed in the 1999.5-2002 time range, with the largest amplitude falling around a period of $31 \mathrm{~d}$.

The periods found by means of the Scargle periodogram and the PDM techniques for the fifteen TSI and SSI subsets are listed in Table 1 in Cols. 3-6 and 7-10, respectively. While the Scargle periodogram retrieves a period close to the solar synodic period up to mid 1997, the PDM technique proves useful up to mid 1998 and possibly up to mid 1999, if the failure for the sixth and the seventh subsets is attributed to the large data gap in mid 1998. However, during the late 1999-2002 interval, i.e., close to the maximum activity of cycle 23 , no reliable determination of the rotation period of the Sun is possible either from the TSI modulation or from each of the SSI modulations.

Therefore, any modelling of the rotational modulation of the TSI and the SSIs during the 1996-2002 period must assume the rotation period as an additional free parameter, the best value of which should be determined with the method described in the next section.

\section{The model of the solar rotational modulation}

To model the simultaneous irradiance variations in the bolometric and optical passbands we adopt the approach of Paper I, originally introduced by Oster et al. (1982) and 
Table 1. The period of the rotational modulation for each of the subsets of 158 days obtained by dividing the entire TSI and SSI time series into 15 time intervals, as determined by means of the Scargle periodogram and the Phase Dispersion Minimization (PDM) technique, respectively.

\begin{tabular}{cccccccccc}
\hline \hline MJD & Year & \multicolumn{3}{c}{$P_{\text {rot }}($ days $)-$ Scargle } & \multicolumn{4}{c}{$P_{\text {rot }}($ days $)-$ PDM } \\
(HJD - 2 400 000) & & TSI & 402 & 500 & 862 & TSI & 402 & 500 & 862 \\
\hline 50259.77 & 1996.57 & 27.7 & 28.3 & 27.3 & 27.5 & 27.2 & 27.1 & 27.1 & 27.3 \\
50417.77 & 1997.00 & 27.6 & 26.1 & 25.7 & 27.9 & 27.8 & 27.1 & 26.9 & 27.8 \\
50575.77 & 1997.44 & 27.9 & 27.4 & 27.0 & 27.7 & 27.9 & 26.7 & 26.7 & 27.7 \\
50733.77 & 1997.87 & 32.6 & 31.6 & 28.4 & 31.6 & 27.5 & 27.5 & 27.5 & 28.1 \\
50891.77 & 1998.30 & 27.0 & 27.0 & 26.8 & 27.1 & 27.3 & 25.8 & 25.6 & 27.1 \\
51049.77 & 1998.73 & 23.1 & 28.4 & 33.4 & 23.5 & 33.5 & 28.5 & 33.5 & 23.5 \\
51207.77 & 1999.16 & 22.5 & 22.8 & 23.7 & 22.4 & 22.3 & 30.6 & 30.7 & 33.1 \\
51365.77 & 1999.60 & 23.3 & 23.3 & 32.5 & 23.1 & 26.2 & 26.1 & 26.3 & 23.2 \\
51523.77 & 2000.03 & 33.2 & 33.1 & 33.4 & 33.0 & 33.5 & 33.4 & 33.5 & 33.4 \\
51681.77 & 2000.46 & 28.4 & 28.5 & 28.7 & 30.4 & 20.4 & 20.8 & 20.4 & 30.0 \\
51839.77 & 2000.90 & 25.4 & 24.8 & 24.9 & 25.1 & 25.4 & 25.2 & 25.2 & 25.2 \\
51997.77 & 2001.33 & 24.0 & 24.2 & 24.7 & 24.0 & 24.2 & 24.3 & 24.4 & 24.2 \\
52155.77 & 2001.76 & 24.2 & 24.3 & 24.5 & 24.4 & 33.5 & 24.1 & 33.5 & 33.5 \\
52313.77 & 2002.19 & 24.8 & 24.0 & 24.8 & 24.8 & 20.3 & 20.3 & 20.3 & 20.3 \\
52471.77 & 2002.63 & 30.3 & 28.8 & 25.6 & 30.3 & 30.0 & 28.1 & 28.1 & 30.0 \\
\hline
\end{tabular}

Sofia et al. (1982). It is based on the following equation as introduced in Paper I:

$$
\begin{array}{r}
S-S_{0}=S_{0} C \sum_{i: \mu_{i} \geq 0} A_{i} \mu_{i}\left[a_{p}+b_{p} \mu_{i}+c_{p} \mu_{i}^{2}\right] \times \\
\left\{\left[c_{\mathrm{s}}-1\right]+Q\left[c_{f}+c_{f}^{\prime} \mu_{i}-1\right]\right\}+S_{r}
\end{array}
$$

where $S$ is the solar irradiance in the given passband, $S_{0}$ its reference value corresponding to the unperturbed Sun, $C$ a normalization coefficient, $\mu_{i} \equiv \cos \psi_{i}$, with $\psi_{i}$ the angle between the normal to the $i$ th active region and the line of sight, $A_{i}$ the area of the $i$ th active region, $a_{p}, b_{p}, c_{p}$ the limb-darkening coefficients, $c_{\mathrm{s}}$ the sunspot contrast coefficient, $c_{\mathrm{f}}, c_{\mathrm{f}}^{\prime}$ the facular contrast coefficients, $Q$ the ratio of the area of the faculae to that of the sunspots and $S_{r}$ the uniformly distributed background term. The limb-darkening and the contrast coefficients appearing in Eq. (1), as well as the unperturbed irradiance $S_{0}$ and the uniformly distributed background term $S_{r}$, now depend on the passband. On the other hand, the areas of the active regions $A_{i}$, their longitudes and latitudes and the parameter $Q$, are assumed independent of the passband. Moreover, the rotation period $P$ of the active region pattern is a free parameter of the model whereas the inclination of the solar rotation axis is given (cf. Paper I).

\subsection{Photometric parameters}

The coefficients of the quadratic limb-darkening laws for the unperturbed photosphere in the SSI and bolometric passbands are computed by means of the LTE atmospheric models of Kurucz (2000) assuming for the Sun an effective temperature $T_{\text {unp }}=5780 \mathrm{~K}$ and a gravity $\log g=4.44$. The transmittance profiles of the sunphotometer passbands are obtained from the official VIRGO web site $^{3}$ (cf. Figs. 1 and 7 in

\footnotetext{
${ }^{3}$ http://virgo.so.estec.esa.nl/
}

Table 2. The quadratic limb-darkening coefficients for the unperturbed solar photosphere for the bolometric and the three sunphotometer passbands, respectively.

\begin{tabular}{cccc}
\hline \hline Passband & $a_{\mathrm{p}}$ & $b_{\mathrm{p}}$ & $c_{\mathrm{p}}$ \\
\hline TSI & 0.3722 & 0.9066 & -0.2788 \\
$402 \mathrm{~nm}$ & 0.1404 & 0.9521 & -0.0925 \\
$500 \mathrm{~nm}$ & 0.2282 & 1.0410 & -0.2692 \\
$862 \mathrm{~nm}$ & 0.4538 & 0.8791 & -0.3330 \\
\hline
\end{tabular}

Fligge et al. 1998). The limb-darkening coefficients are listed in Table 2 and their values are in satisfactory agreement with the measured ones, as listed by Pierce (2000).

The sunspot contrast coefficient in a given passband is computed from the ratio of the specific intensities of the spotted and the unspotted photospheres at the center of the solar disk, as derived from Kurucz's models. The effective temperature of the spotted photosphere is given by $T_{\mathrm{s}}=T_{\text {unp }}+\Delta T_{\mathrm{s}}$, where the spot temperature difference $\Delta T_{\mathrm{s}}$ is a free parameter of our model, while the gravity of the spotted photosphere is assumed to be equal to that of the unperturbed photosphere. The variation of the contrast from the disk center to the limb is of only a few percent, which supports our assumption of a constant contrast in a given passband. The spot contrasts are plotted versus the temperature difference $\Delta T_{\mathrm{s}}$ for the TSI and SSI passbands in Fig. 2.

The Kurucz atmospheric models adopted in the present study yield contrast differences $<1 \%$ in the bolometric band and $<3 \%$ in the SSI passbands with respect to the previous ones (Kurucz 1991) adopted by Fligge et al. (1998). The standard sunspot bolometric contrast adopted in the calculation of the Sunspot Photometric Index, i.e. $\alpha=0.33$ (see 


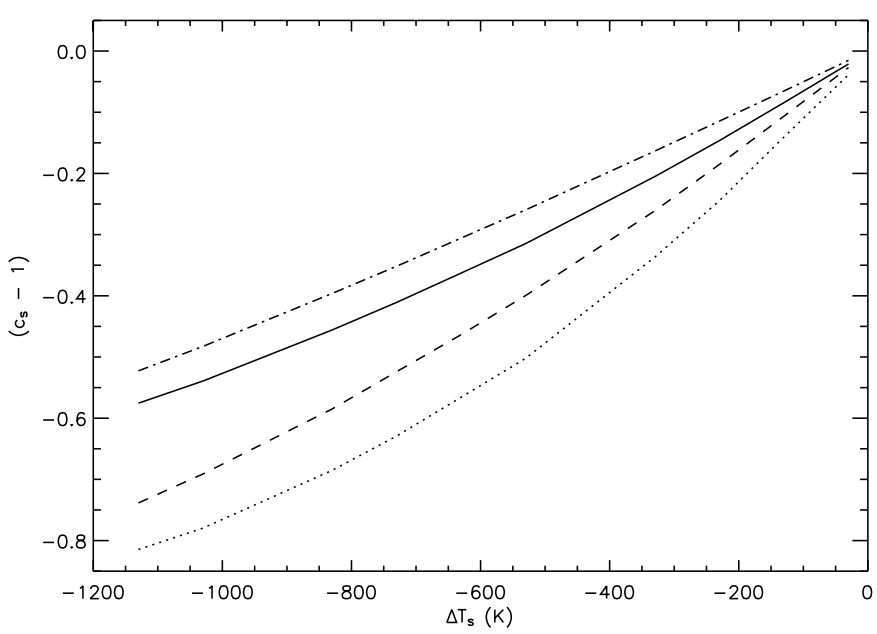

Fig. 2. The sunspot contrasts adopted in our model $\left(c_{\mathrm{s}}-1\right)$ versus the temperature difference of the sunspots with respect to the unperturbed photosphere $\Delta T_{\mathrm{s}}$. Different linestyles refer to different passbands: TSI - solid; $402 \mathrm{~nm}$ - dotted; $500 \mathrm{~nm}$ - dashed; $862 \mathrm{~nm}$ dash-dotted.

Chapman et al. 1994, for details), corresponds to a temperature deficit $\Delta T_{\mathrm{s}} \simeq-560 \mathrm{~K}$ in our model.

The monochromatic facular contrast is computed according to Chapman \& McGuire (1977): $\Delta I_{\lambda} / I_{\lambda} \propto \lambda^{-1}\left(\Delta T_{\mathrm{f}} / T_{\text {unp }}^{2}\right)$, where $\Delta T_{\mathrm{f}}$ is the facular excess temperature with respect to the unperturbed photosphere and $\lambda$ is the wavelength. It is averaged over the SPM passbands to give the contrast factors appearing in Eq. (1). The bolometric contrast is assumed equal to the contrast averaged over the spectral interval between $350 \mathrm{~nm}$ and $1000 \mathrm{~nm}$, according to the approximation introduced by Foukal et al. (1991). The contrasts are plotted versus the facular temperature excess $\Delta T_{\mathrm{f}}$ for the four passbands in Fig. 3. A comparison of our facular contrasts for $\Delta T_{\mathrm{f}}=100 \mathrm{~K}$ with those by Fligge et al. (1998) shows differences less than $15 \%$ in the bolometric passband and less than $25 \%$ in the SPM passbands, as expected on the basis of our simplified treatment of facular irradiance.

For the sake of simplicity, the angular dependence of the facular contrast is assumed to be linear with zero contrast at the disk center $(\mu=1)$ and maximum contrast at the limb $(\mu=0)$, which implies $c_{\mathrm{f}}^{\prime}=-c_{\mathrm{f}}$ in each passband in Eq. (1). As a matter of fact, Lawrence (1988) proposed that a better approximation to the center-to-limb dependence of the monochromatic facular contrast is provided by a linear regression versus $\mu^{-1}$, but other authors suggested different dependences (cf. Unruh et al. 1999). In any case, departures from our approximation are significant only for $0<\mu<0.3$ and the deviations do not exceed $10-20 \%$ of the irradiance variations because the projected area $A_{i} \mu_{i}$ in Eq. (1) is made small by the foreshortening effect. Moreover, the observations show that the contrasts of faculae and, to a lesser extent, of the sunspots vary significantly among active regions (Chapman 1987; Chapman et al. 1994) and systematic changes with the phase of the solar cycle are also possible (Albregtsen et al. 1984). $Q$ was fixed at the same value as adopted in Paper I, i.e., $Q=10$.

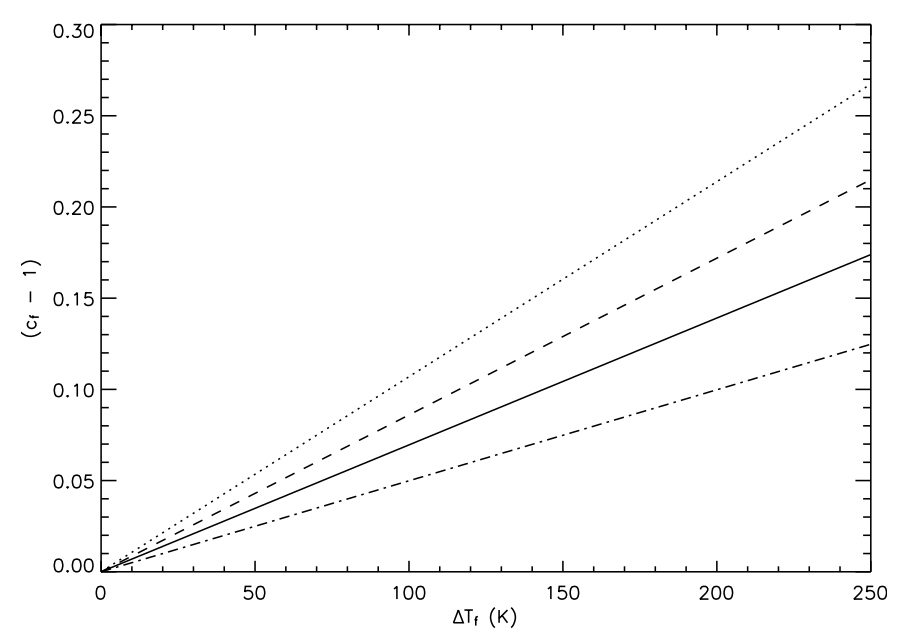

Fig. 3. The facular contrasts adopted in our model $\left(c_{\mathrm{f}}-1\right)$ versus the temperature excess of the faculae with respect to the unperturbed photosphere $\Delta T_{\mathrm{f}}$. Different linestyles refer to different passbands: TSI solid; $402 \mathrm{~nm}$ - dotted; $500 \mathrm{~nm}$ - dashed; $862 \mathrm{~nm}$ - dash-dotted.

The uniformly distributed background is accounted for by means of the terms $S_{r}(\lambda)$ in our model which now depends on the wavelength $\lambda$. The contrast of the small flux tubes of the network is different from that of the larger flux tubes found in facular regions (Ortiz et al. 2002), hence we decided to estimate the spectral distribution of the network term adopting a simple trial-and-error approach by letting only the bolometric background term $S_{r}$ (bol) vary and correcting the guess values of the ratios $S_{r}(\lambda) / S_{r}$ (bol) until a good fit was obtained for an extended interval of data close to the solar minimum. In this way, we found that the best fit was obtained for the values: $S_{r}(402) / S_{r}(\mathrm{bol})=0.650, S_{r}(500) / S_{r}(\mathrm{bol})=0.327$ and $S_{r}(862) / S_{r}(\mathrm{bol})=0.330$, respectively, where the SSI bands are indicated by their central wavelengths. In other words, only one free parameter was left in our multiwavelength model to fit the uniformly distributed background, i.e., $S_{r}$ (bol). The fixed values of the ratios given above proved adequate also during the rising, the maximum and the declining phases of activity cycle 23 and were held fixed in our modelling.

The value of the reference irradiance $S_{0}(\lambda)$, i.e., the solar irradiance at wavelength $\lambda$ without active region and uniform network contribution, is unknown in a stellar-like approach. $S_{0}(\lambda)$ is the value with respect to which the model irradiance in the passband $\lambda$ must be normalized before fitting the observed variations. In modelling stellar wide-band light curves it is customary to adopt as a reference value the flux corresponding to the brightest magnitude of the star in the considered time series, i.e., at the minimal coverage of the stellar disk by cool spots (cf. e.g., Messina et al. 1999). In the analysis presented in Paper I, we could adopt the maximum of the TSI along its entire time series because the TSI data were corrected for the long-term instrumental trends. In the present analysis, the SSI oscillations introduced by the inaccurate detrending of the long-term instrumental effects make it impossible to follow the same procedure. After a series of tests, and considering that the residual long-term trends can induce variations of the order of $100 \mathrm{ppm}$ on time scales of 15-20 d in the $402 \mathrm{~nm}$ 
channel, especially during the solar maximum phases, we decided to split the time series into subsets of length $14 \mathrm{~d}$ and normalize each one independently from the others. The value of $S_{0}(\lambda)$ for each SSI subset was chosen to be equal to the value observed simultaneously with the maximum of the TSI during that 14-d time span. This approach allowed us to obtain always a good fit, minimizing the residuals up to the accuracy of the individual data, but at the cost of losing all the information on the long-term trends related to the evolution of the activity complexes and the solar cycle. We point out that this limitation is not a drawback of our modelling approach but comes unavoidably from the fact that the long-term variations of the SSIs are contaminated by the unknown degradation of the sunphotometers (cf. Sect. 2).

\subsection{Free model parameters and time series fitting}

The best values of our free-model parameters can be found by minimizing the $\chi^{2}$ statistics, defined as:

$$
\chi^{2}=\frac{1}{4} \sum_{k=1}^{4} \frac{1}{M} \sum_{j=1}^{M} \frac{\left[S_{\text {obs }}\left(t_{j}, \lambda_{k}\right)-S\left(t_{j}, \lambda_{k}\right)\right]^{2}}{\sigma\left(\lambda_{k}\right)^{2}},
$$

where the index $k$ specifies the passband of central wavelength $\lambda_{k}, M$ is the number of simultaneous observations in the considered interval of time, $S_{\text {obs }}\left(t_{j}, \lambda_{k}\right)$ the observed value of the solar irradiance in the $k$ th passband at time $t_{j}, S\left(t_{j}, \lambda_{k}\right)$ the value of the irradiance in the $k$ th passband evaluated from Eq. (1) at the same time, and $\sigma\left(\lambda_{k}\right)$ the standard deviation of the measurements in the $k$ th passband as computed from the relative values given in Sect. 2 .

The allowed interval of variation of each parameter or of a combination of independent parameters for a given confidence level can be estimated by means of the procedures described by Lampton et al. (1976). Specifically, we shall apply the ratio-of-variance method that is independent of the values of the variance of the individual data and is therefore useful for avoiding the effects of the short-term fluctuations of the solar irradiance. The variance of a fit obtained with a given set of parameters is defined as: $S=4 M \chi^{2}$, where $\chi^{2}$ is given by Eq. (2). The best values of the parameters correspond to the minimum of $S$, i.e., $S_{\min }$. The confidence region for the free parameters with significance level $\alpha$ is the parameter range giving $S<S_{L}$, where:

$S_{L}=S_{\min }\left[1+\frac{p}{4 M-p} F(p, 4 M-p, \alpha)\right]$,

where $4 M$ is the number of data, $p$ the number of free parameters in the model and $F$ is the Fisher-Snedecor distribution (Wadsworth 1990). By considering fixed values of $\Delta T_{\mathrm{s}}$ and $\Delta T_{\mathrm{f}}$ (see below) and assuming three model active regions as in Paper I, the number of free parameters $p=13$ for each 14-d data subset.

Note that our model parameters are not completely independent of each other. Similar monochromatic variations can be produced by changing the projected area of an active region or the contrast coefficients because they are inversely correlated. The contrast coefficients depend almost linearly on $\Delta T_{\mathrm{s}}$ and $\Delta T_{\mathrm{f}}$ (cf. Figs. 2 and 3), leading to a correlation of these parameters with the projected area of an active region. In turn, the projected area depends on the latitude of an active region. In the case of the Sun, it is difficult to discriminate among regions at different latitudes because the inclination of the rotation axis is close to $90^{\circ}$, implying that the duration of the transit of a surface feature does not depend significantly on its latitude, but on the rotation period $P$ of our star which is an additional free parameter of the model.

Due to the finite data accuracy and the short-term intrinsic fluctuations, this implies that the problem of fitting our model to the simultaneous TSI and SSI variations is ill-conditioned. In particular, a non-unique and unstable solution is usually found when the values of the temperature differences $\Delta T_{\mathrm{s}}$ and $\Delta T_{\mathrm{f}}$ are included among the free parameters. Therefore the temperature differences $\Delta T_{\mathrm{s}}$ and $\Delta T_{\mathrm{f}}$ must be kept fixed for each fit. Moreover, a numerical instability may still arise in the fitting of a given data set because a linear combination of active region longitude and angular velocity appears in Eq. (2) of Paper I. Therefore we have always fitted each 14-d data subset by fixing a test value for the rotation period and then finding the best values of the active region areas and coordinates that minimized the $\chi^{2}$ as explained in Paper I. Then the rotation period $P$ was iteratively adjusted in the interval $23.0-33.5 \mathrm{~d}$ by performing successive fitting to locate the absolute minimum of the $\chi^{2}$ by means of a golden section algorithm (Press et al. 1992).

Our procedure of fitting the geometrical parameters of the active regions only for fixed values of $\Delta T_{\mathrm{s}}, \Delta T_{\mathrm{f}}$ and $P$ and then minimizing the $\chi^{2}$ with respect to $P$, eliminates most of the instability problems. Moreover, other sources of instability can be controlled by setting limits on the active region relative area and the variation of the uniform background term.

Specifically, the depth of the largest dips of the TSI and SSI time series are used to fix limits for the spot area: $0 \leq$ $A_{i} \leq 8.2 \times 10^{-4}$; while the variation of $S_{r}(\mathrm{bol})$ is constrained by adopting the same interval as in Paper I: $-1.5 \times 10^{-4} \leq$ $S_{r}(\mathrm{bol}) / S_{0}(\mathrm{bol}) \leq 1.5 \times 10^{-4}$. This sets corresponding limits on the variations of $S_{r}\left(\lambda_{k}\right)$ since the ratios $S_{r}\left(\lambda_{k}\right) / S_{r}($ bol $)$ are held fixed.

\section{Results}

The determination of $\Delta T_{\mathrm{s}}$ and $\Delta T_{\mathrm{f}}$ requires the selection of a data subset during which the irradiance variations are dominated by the rotational modulation of one stable active region. When the active region evolution becomes important, or when several active regions are simultaneously affecting the irradiance, the trade-off among the model parameters makes it impossible to obtain a unique determination of the contrasts and hence of $\Delta T_{\mathrm{s}}$ and $\Delta T_{\mathrm{f}}$ (cf. Sect. 3.2). Therefore, we considered the TSI and SSI time series ranging from 16 July 1996 to 17 August 1996, encompassing the transit of the single active region NOAA 7981 when the irradiance changes were dominated by the modulation of its visibility. The same data subset was selected by Eker et al. (2003) to derive the sunspot effective temperature in the framework of a stellar-like approach.

The isocontours of the ratio $S / S_{\text {min }}$ are plotted in Fig. 4 versus $\Delta T_{\mathrm{s}}$ and $\Delta T_{\mathrm{f}}$, respectively. The minimum value of $S$, 


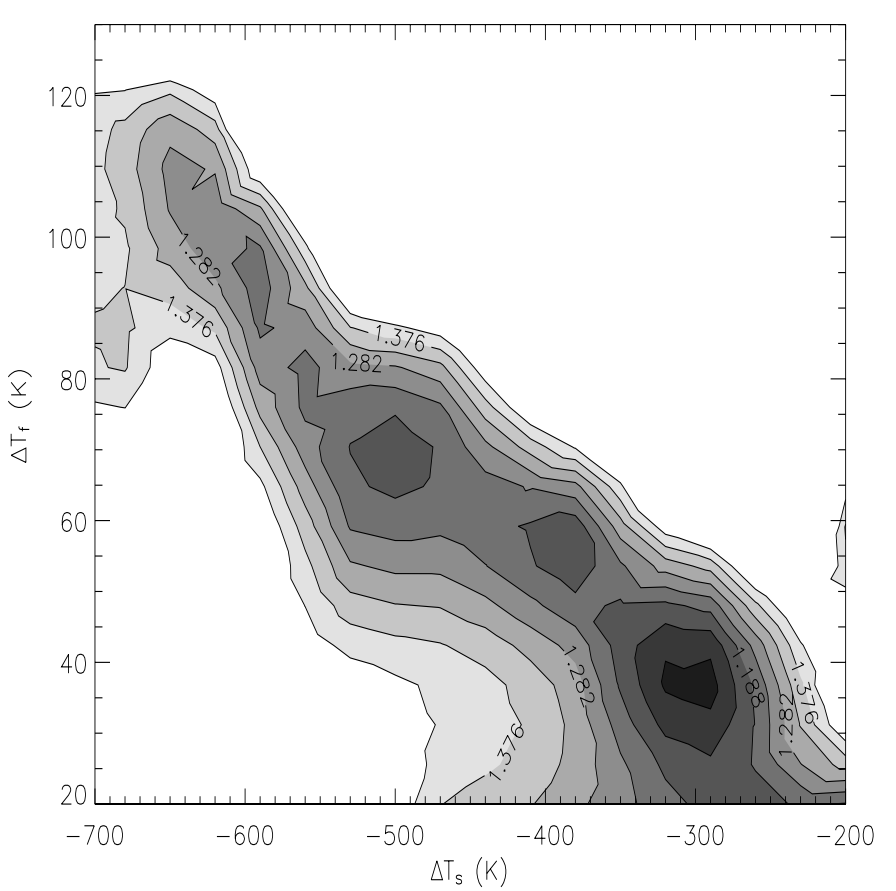

Fig. 4. The isocontour plots of the ratio-of-variance statistics $S / S_{\min }$ versus sunspot temperature deficit $\Delta T_{\mathrm{s}}$ and facular temperature excess $\Delta T_{\mathrm{f}}$ for the data subset ranging from 16 July 1996 to 17 August 1996, close to the minimum of solar activity cycle 23 .

$S_{\text {min }}$, corresponds to an average $\chi^{2}=0.91$ for this data set. The value $S_{L} / S_{\min }$ for a confidence level of 0.01 is 1.075 , indicating that the confidence intervals for the sunspot temperature deficit and the facular temperature excess are $-300 \pm 30 \mathrm{~K}$ and $40 \pm 10 \mathrm{~K}$, respectively, in agreement with the sunspot temperature deficit estimated by Eker et al. (2003). Note, however, the extended trade-off between the values of $\Delta T_{\mathrm{s}}$ and $\Delta T_{\mathrm{f}}$ for isocontours encompassing larger values of $S / S_{\text {min }}$, which indicates that the solution may become non-unique for larger values of the measurement errors. For the sake of completeness, we sampled the $\Delta T_{\mathrm{s}}-\Delta T_{\mathrm{f}}$ parameter space also with models assuming one or two active regions, respectively. For one active region, the best fit turned out to be inadequate, with an average $\chi^{2}=6.79$ that implied that no meaningful temperature determination could be made on the basis of this oversimplified model. The model with two active regions yielded two comparable relative minima, one with $\chi^{2}=1.37$ for $\Delta T_{\mathrm{s}}=-680 \pm$ $40 \mathrm{~K}$ and $\Delta T_{\mathrm{f}}=40 \pm 10 \mathrm{~K}$ and the other with $\chi^{2}=1.42$ for $\Delta T_{\mathrm{s}}=-430 \pm 30 \mathrm{~K}$ and $\Delta T_{\mathrm{f}}=40 \pm 10 \mathrm{~K}$.

The application of the same approach to other data subsets led to non-unique solutions for $\Delta T_{\mathrm{s}}$ and $\Delta T_{\mathrm{f}}$ even close to the solar minimum, because of the sizeable active region evolution and the simultaneous presence of several active regions affecting the flux modulations. Therefore, in order to fit the entire data sequence, we started with the values of $\Delta T_{\mathrm{s}}$ and $\Delta T_{\mathrm{f}}$ obtained by the analysis of the above data subset (16 July-17 August 1996) and varied them by steps of $20 \mathrm{~K}$ and $10 \mathrm{~K}$, respectively, using a trial-and-error approach until a satisfactory fit to the entire data sequence (7 April 1996-30 November 2002) was obtained. In such a way, we found that $\Delta T_{\mathrm{s}}=-380 \mathrm{~K}$ and $\Delta T_{\mathrm{f}}=60 \mathrm{~K}$ provided

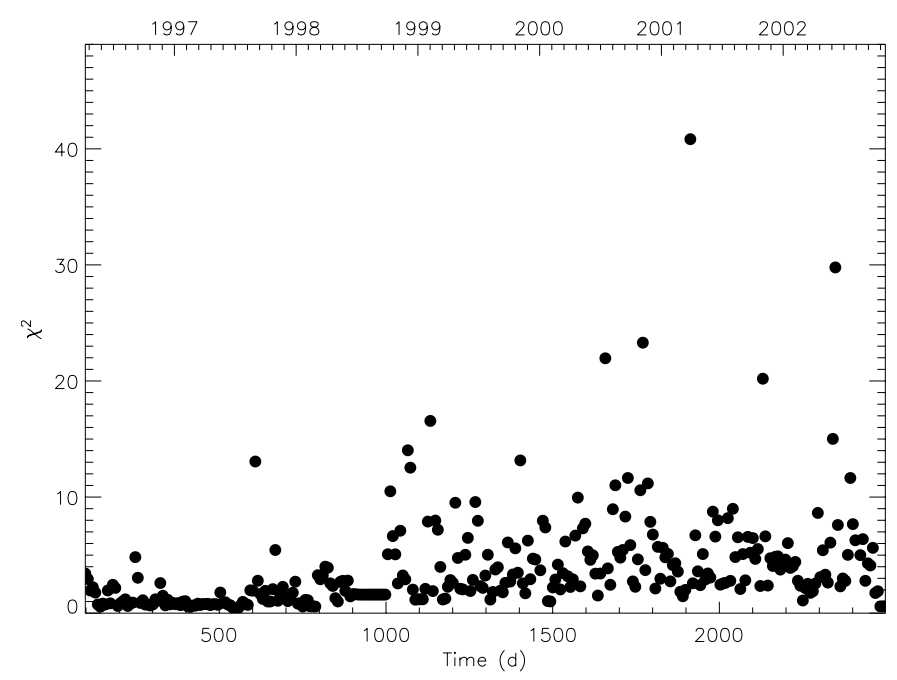

Fig. 5. The $\chi^{2}$ of the individually fitted data subsets of length 14 days versus time computed according to Eq. (2). The time is indicated in days counting from 1st January 1996 on the lower scale and in years on the upper scale.

us with the best fit to the entire data set with the $\chi^{2}$ values of the individual 14-d data subsets plotted in Fig. 5. The $\chi^{2}$ values are close to unity, except in $\approx 30 \%$ of the cases. There is a systematic increase of the values of $\chi^{2}$ toward the maximum of activity that can be attributed to a simultaneous increase of the irradiance variability, on time scales ranging from a few hours to a few days that cannot be adequately fitted with our rotational modulation model.

We note that our sunspot temperature deficit is smaller than the value of Fligge et al. (1998) and those adopted in the PSI calculations (Chapman et al. 1994) because the areas of our model active regions are systematically larger than those observed, especially during solar minimum, which requires a lower contrast to fit the variations. Similarly, our lower facular temperature excess with respect to that of Fligge et al. (1998) can be qualitatively explained by our systematically larger facular areas.

We obtained an improvement of the fit for the data subset ranging from 6 November to 23 December 1996 by adopting $\Delta T_{\mathrm{s}}=-630 \mathrm{~K}$ and $\Delta T_{\mathrm{f}}=120 \mathrm{~K}$ with a reduction of the overall $\chi^{2}$ by $\sim 20 \%$. This result agrees with the model temperatures of Fligge et al. (1998), but such values of $\Delta T_{\mathrm{s}}$ and $\Delta T_{\mathrm{f}}$ yielded poorer fits during other time intervals and could not be adopted for the overall best fit.

In Figs. 6 and 7 we display the best fits to some selected data subsets, obtained with $\Delta T_{\mathrm{s}}=-380 \mathrm{~K}$ and $\Delta T_{\mathrm{f}}=60 \mathrm{~K}$. They encompass intervals close to the minimum and the maximum of solar cycle 23, respectively, and include some of the data subsets previously fitted by Fligge et al. (2000), Eker et al. (2003) and Krivova et al. (2003) with different approaches. As in Paper I, the value of the fit for a given measurement is computed with the parameters determined by the best fit of the 14-d data subset whose midpoint is the closest preceding the measurement. Thus the time interval between a given measurement and the nominal epoch of the best fit parameters is always smaller than $7.0 \mathrm{~d}$. The best fits show deviations ranging 

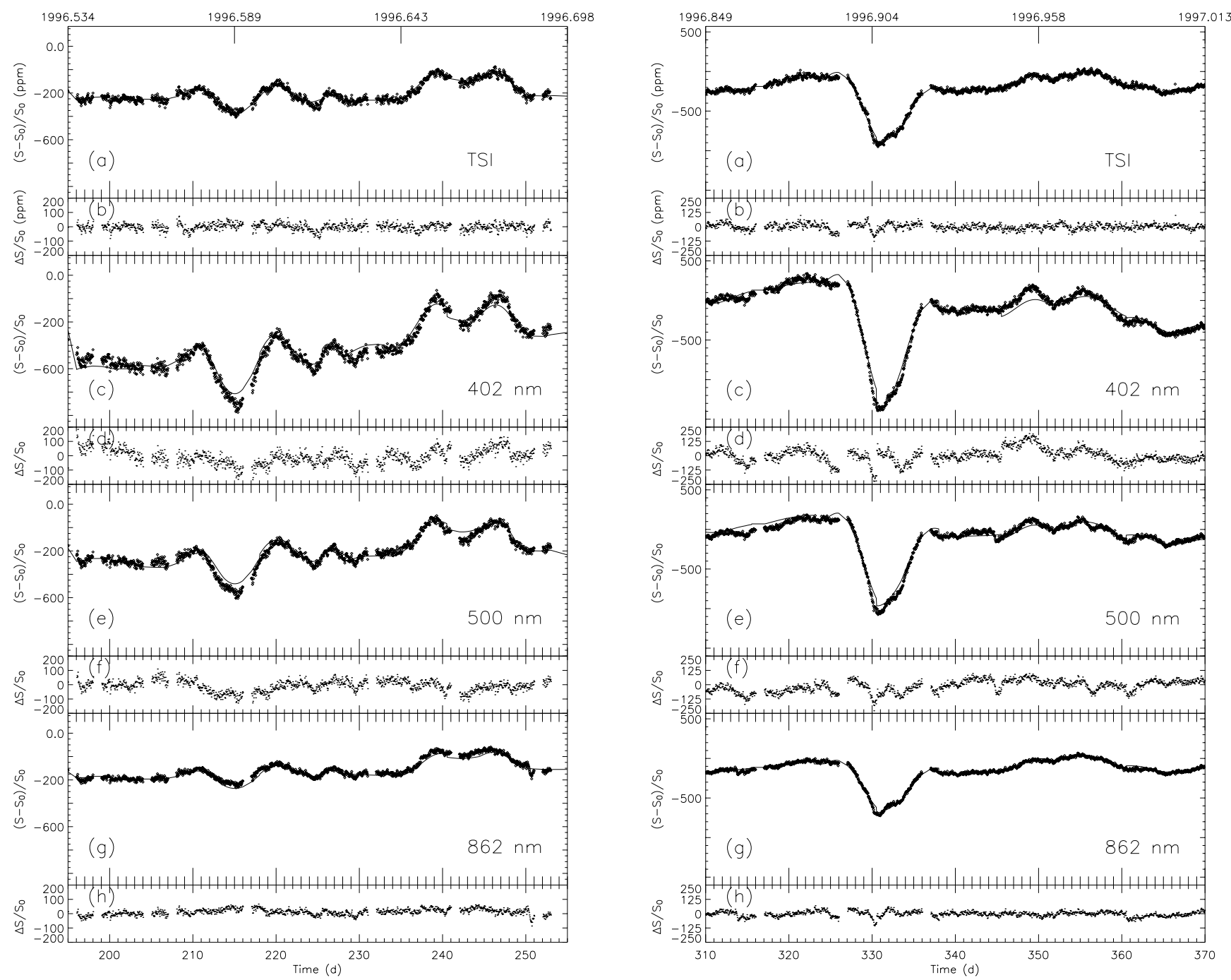

Fig. 6. Two subsets of the TSI and SSI time series during the minimum of solar cycle 23 (open diamonds) with their respective best fits (solid lines) are plotted in panels a), c), e) and $\mathbf{g}$ ) for the labelled passbands in the left and the right columns, respectively. The model is usually embedded into the data sequence except when data gaps are present. The residuals are plotted in the corresponding lower panels b), d), f) and h), respectively. The time is indicated in days from 1st January 1996 on the lower scale and in years on the upper scale. The data subsets range from 13 July 1996 to 11 September 1996 on the left panels, including the intervals previously analysed by Eker et al. (2003) and Fligge et al. (2000, Fig. 6), and from 5 November 1996 to 4 January 1997 on the right panels, previously analysed by Fligge et al. (2000, Fig. 7), respectively.

from $\sim 50 \mathrm{ppm}$ for the TSI to $\sim 200 \mathrm{ppm}$ for the $402 \mathrm{~nm}$ passband at solar minimum, rising to $\sim 150 \mathrm{ppm}$ for the TSI and up to $\sim 300-350 \mathrm{ppm}$ for the $402 \mathrm{~nm}$ passband close to the solar maximum.

The performance of our modelling approach in the frequency domain can be quantitatively estimated by means of standard bivariate analysis techniques (cf. e.g., Priestley 1981). Specifically, we may assume that the relationship between the observed time series and the fitting time series is of the form:

$S_{\mathrm{obs}}\left(t_{j}, \lambda_{k}\right)=\sum_{l=-\infty}^{\infty} g_{\lambda_{k}}(l) S\left(t_{j-l}, \lambda_{k}\right)+N\left(t_{j}, \lambda_{k}\right)$

where $S_{\text {obs }}$ and $S$ are the observed and the best fit value of the solar irradiance at time $t_{j}$ in the passband $\lambda_{k}, g_{\lambda_{k}}(l)$ is the impulse response for a delay of $l$ time steps and $N_{j}\left(t_{j}, \lambda_{k}\right)$ is the noise at time $t_{j}$ in the given passband. The relationship between the two time series is expressed by the transfer functions $\Gamma_{\lambda_{k}}(\omega) \equiv \sum_{l=-\infty}^{\infty} g_{\lambda_{k}}(l) \exp (-i \omega l)$, where $i=\sqrt{-1}$. An ideal model would have $g_{\lambda_{k}}(0)=1$ and $g_{\lambda_{k}}(l)=0, \forall l \neq 0$, i.e., a gain $\left|\Gamma_{\lambda_{k}}(\omega)\right|=1$ and a phase spectrum $\arg \left\{\Gamma_{\lambda_{k}}(\omega)\right\}=0, \forall \omega$. Moreover, bivariate analysis provides us with another quantity that measures the degree of linear correlation of the two time series at frequency $\omega$, i.e., their coherency. When the square of the modulus of the coherency is close to unity at all frequencies, we would expect to obtain a close linear fit between the two series.

The gain, the phase spectrum and the squared modulus of the coherency for our model are plotted in Fig. 8 for the time interval ranging from 1996.25 to 1997.75 , i.e., close to the minimum of solar cycle 23, and for the interval from 1999.5 to 2001 , encompassing the maximum of cycle 23 , respectively. 

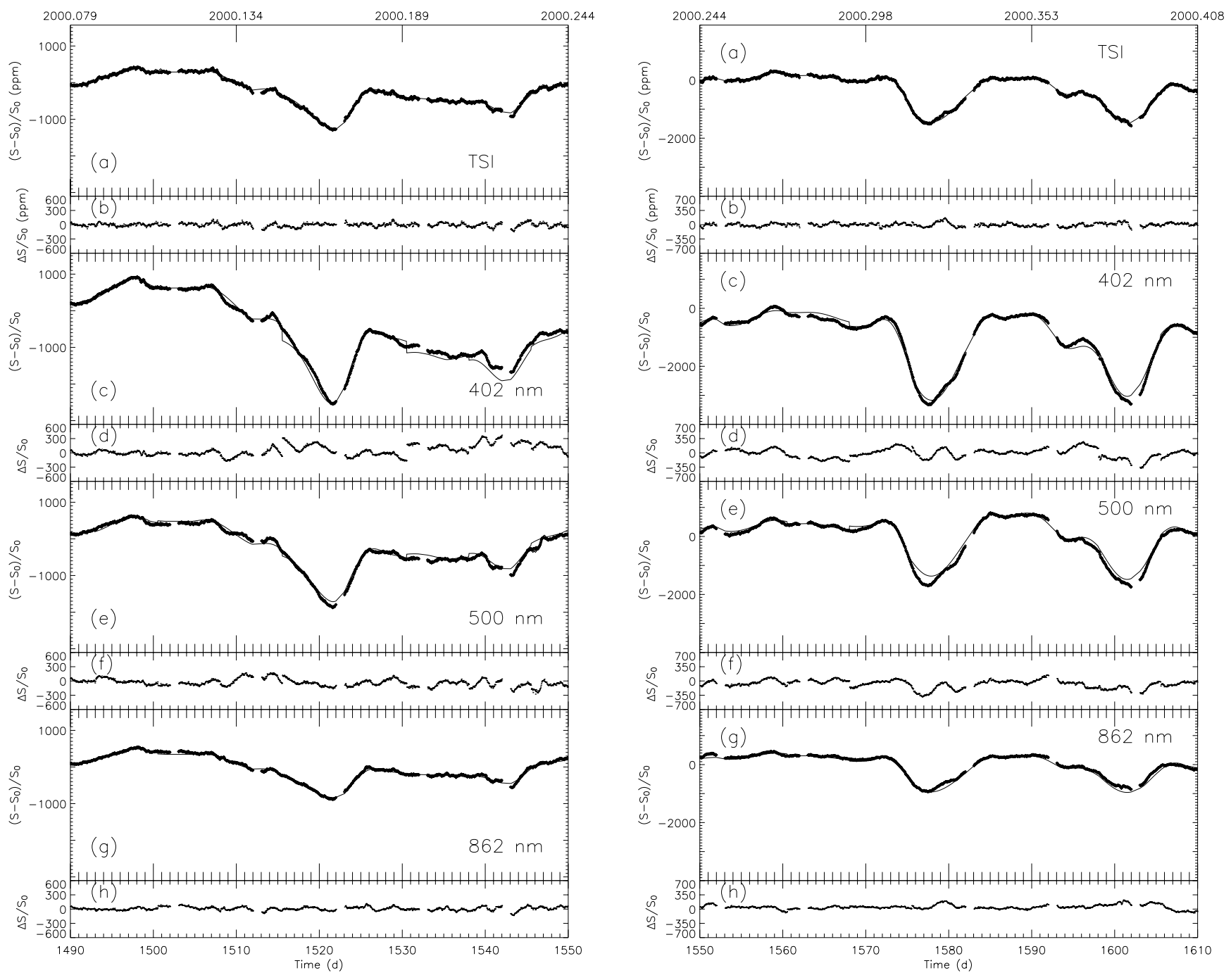

Fig. 7. The same as Fig. 6 for two data subsets close to the maximum of solar cycle 23, ranging from 29 January 2000 to 29 March 2000 in the left panels, and from 29 March 2000 to 28 May 2000 in the right panels, respectively. These time series were previously modelled by Krivova et al. (2003, Fig. 3 right panels).

The model time series provide a good fit to the observed time series in the four passbands for $\omega \leq 0.2\left(\mathrm{~d}^{-1}\right)$, i.e., for time scales longer than 5.0 days during the activity minimum phase, with a significant degradation of the performance toward increasing frequencies, likely to be due to the irradiance fluctuations produced by ephemeral active regions with typical lifetimes of 2-3 days (Harvey 1993). During the maximum of activity, the best performance is obtained for frequencies $\omega \leq 0.25\left(\mathrm{~d}^{-1}\right)$, i.e., time scales longer than 4 days, but the degradation of the performance towards higher frequencies is less pronounced and the squared modulus of the coherency stays at about 0.8 for the $402 \mathrm{~nm}$ band up to $\omega \sim 1.0\left(\mathrm{~d}^{-1}\right)$. This suggests that the variability of the solar irradiance close to the maximum of activity is dominated by long-lived active regions affecting predominantly the TSI and the longer optical wavelengths. Moreover, during the maximum activity interval, our model gives a better reproduction of the phase shifts due to the active region growth and decay than during the interval of minimum activity, as witnessed by the smaller dispersion of the phase spectrum around the zero values. This indicates that the typical lifetime of the surface inhomogeneities is comparable to the typical time scale we assumed for changes of the active region configuration, i.e., 7 days in the present model. The determinations of the rotation period $P$ and of the longitude of the active regions are not significantly improved by the simultaneous fitting of the TSI and SSI data. For a discussion of such aspects and the long-term parameter variations related to the 11-yr cycle, we refer the interested reader to Paper I.

\section{Discussion and conclusions}

The application of stellar-like approaches for modelling the solar rotational modulation is not new (cf. Paper I, and references therein). However, this is the first time, to our knowledge, that total and optical multiband irradiance data are simultaneously modelled without any knowledge of the distribution of active regions on the solar disk that can help to constrain the free parameters (cf. Eker et al. 2003). The direct approach, i.e., the modelling of the solar irradiance variations in the TSI and SSI passbands starting from the maps of the surface magnetic 

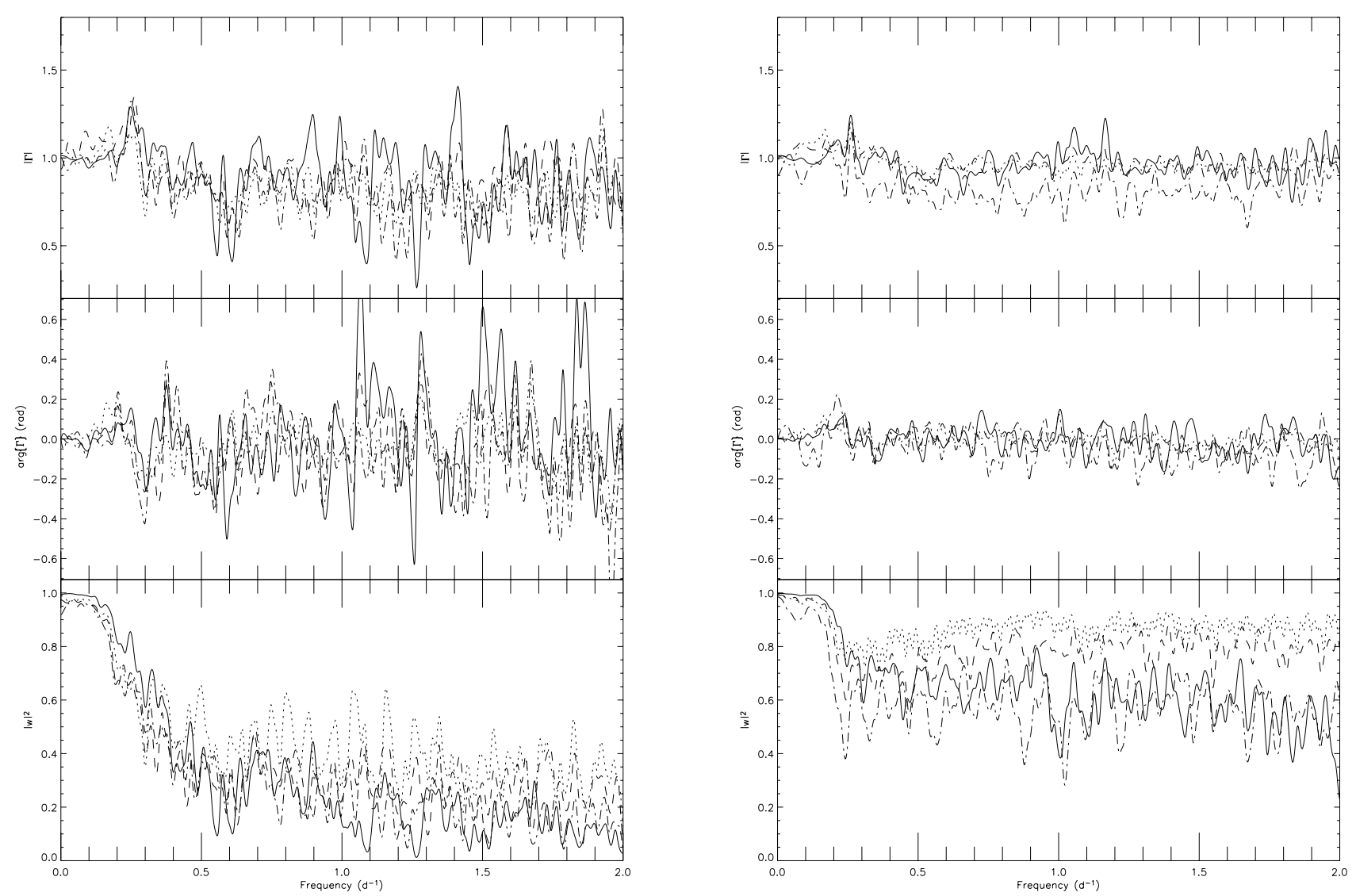

Fig. 8. From the upper to the bottom left panels: the gain spectrum $|\Gamma|$, the phase spectrum $\arg \{\Gamma\}$ and the square of the modulus of the coherency $|w|^{2}$ versus frequency for our model time series versus the corresponding observed time series during the interval ranging from 1996.25 to 1997.75. Different linestyles mark the four passbands: solid - TSI; dotted - $402 \mathrm{~nm}$; dashed - $500 \mathrm{~nm}$; dash-dotted - $862 \mathrm{~nm}$. From the upper to the bottom right panels: the same as the left panels for the interval ranging from 1999.5 to 2001.0.

field and brightness distributions, was pursued by, e.g., Fligge et al. (2000) and Krivova et al. (2003).

The simultaneous multiband fits obtained with our model are comparable with the irradiance variations reconstructed by means of the direct approach. The important conclusion that stems from this result is that most of the solar variability in the TSI and SSI passbands is related to the growth and decay of active regions on time scales longer than 5-7 days and to the rotational modulation of their visibility.

The main drawback of our approach is the number of free parameters and the trade-off among them. The rotation period can be safely estimated only during the solar minimum phase. The sunspot temperature deficit and facular temperature excess cannot be derived except for the rare cases in which a very simple and highly stable active region configuration dominates the rotational modulation. This implies that information on the surface distribution of the brightness inhomogeneities is usually necessary to estimate unambiguously sunspot and facular contrasts as well as their area ratio (cf. Eker et al. 2003).

In the case of distant stars, we may get some information only for those objects that rotate fast enough to have rotationally broadened spectral line profiles that allow us to apply the Doppler imaging technique $\left(v \sin i>20 \mathrm{~km} \mathrm{~s}^{-1}\right)$. This may provide us with information on the latitude and longitude of surface brightness inhomogeneities as well as on their temperature (e.g., Strassmeier et al. 2003). Moreover, information on the equatorial rotational velocity and the inclination of the rotation axis can be inferred.

The most important conclusion from the present analysis is that it is possible to model most of the variability of the Sun by means of a simple geometrical model. If the solar-like stars to be monitored by means of the forthcoming space-borne photometric telescopes have the same kind of variability, it will be possible to reduce significantly the impact of magnetic activity on the detection of planetary transits, as well as of the light reflected by close-by giant planets. Our approach will also be helpful for the derivation of accurate light curves of the transit or of the reflected light in order to estimate the planet's radius and albedo (cf. Paper I). A quantitative assessment of the advantages of our techniques for planet search and the characterization of their physical properties is foreseen in forthcoming papers (Barge et al., in preparation).

Acknowledgements. The authors wish to thank the Referee, C. Fröhlich, for a critical reading of the manuscript and useful suggestions and A. Baglin, P. Barge, F. Favata, A. Ortiz and D. Spadaro for stimulating discussions. They also acknowledge R. Ventura, who provided the Fortran code for the Scargle periodogram analysis, and R. Kurucz, who made available to them his stellar atmosphere 
models. The availability of the VIRGO/SoHO data on total solar irradiance and spectral irradiances from the VIRGO Team through PMOD/WRC, (Davos, Switzerland) and of unpublished data from the VIRGO Experiment on board of the ESA/NASA Mission SoHO are gratefully acknowledged.

Research on stellar physics at Catania Astrophysical Observatory of the INAF (Istituto Nazionale di Astrofisica), and at the Dept. of Physics and Astronomy of Catania University is funded by MIUR (Ministero dell'Istruzione, Università e Ricerca) and Regione Sicilia, whose financial support is gratefully acknowledged. The extensive use of computer facilities at the Catania node of the Italian Astronet Network is also gratefully acknowledged.

\section{References}

Aigrain, S., Favata, F., \& Gilmore, G. 2004, A\&A, 414, 1139

Albregtsen, F., Ioras, P. B., \& Maltby, P. 1984, Sol. Phys., 90, 17

Anklin, M., Fröhlich, C., Finsterle, W., Crommelynck, D. A., \& Dewitte, S. 1999, Metrologia, 35, 685

Baglin, A., Auvergne, M., Catala, C., Michel, M., \& COROT Team 2001, ESA-SP, 464, 395

Chapman, G. A. 1987, ARA\&A, 25, 633

Chapman, G. A., Cookson, A. M., \& Dobias, J. J. 1994, ApJ, 432, 403

Chapman, G. A., \& McGuire, T. E. 1977, ApJ, 217, 657

Crommelinck, D., Dewitte, S., \& Chevalier, A. 2004, in preparation

Defay, C., Deleuil, M., \& Barge, P. 2001, A\&A, 365, 330

de Toma, G., White, O. R., Chapman, G. A., et al. 2001, ApJ, 549, L131

Dobson, A. K., Donahue, R. A., Radick, R. R., \& Kadlec, K. L. 1990, The Sixth Cambridge Symp. on Cool Stars, ed. G. Wallerstein, ASP Conf. Ser., 9, 132

Donahue, R. A. 1993, Ph.D. Thesis, New Mexico State Univ.

Donahue, R. A., \& Baliunas, S. L. 1992, ApJ, 393, L63

Donahue, R. A., Dobson, A. K., \& Baliunas, S. L. 1997a, Sol. Phys., 171,191

Donahue, R. A., Dobson, A. K., \& Baliunas, S. L. 1997b, Sol. Phys., 171,211

Eker, Z., Brandt, P. N., Hanslmeier, A., Otruba, W., \& Wehrli, C. 2003, A\&A, 404, 1107

Fligge, M., Solanki, S. K., \& Unruh, Y. C. 2000, A\&A, 353, 380

Fligge, M., Solanki, S. K., Unruh, Y. C., Fröhlich, C., \& Wehrli, Ch. 1998, A\&A, 335, 709

Foukal, P., Harvey, K., \& Hill, F. 1991, ApJ, 383, L89

Foukal, P., \& Lean, J. 1988, ApJ, 328, 347

Foukal, P., Solanki, S., \& Zirker, J. 2000, in Allen's Astrophysical Quantities, the 4th edition, ed. A. N. Cox (Berlin: SpringerVerlag), 364

Fröhlich, C. 2003, Metrologia, 40, 60

Fröhlich, C., Andersen, B. N., Appourchaux, T., et al. 1997b, Sol. Phys., 170, 1

Fröhlich, C., Crommelynck, D., Wehrli, C., et al. 1997a, Sol. Phys., 175,267
Fröhlich, C., \& Finsterle, W. 2001, in Recent Insights into the Physics of the Sun and Heliosphere: Highlights from SOHO and other Space Missions, ed. P. Brekke, B. Fleck, \& J. B. Gurman, Astr. Soc. Pac., IAU Symp., 203, 105

Fröhlich, C., Romero, J., Roth, H., et al. 1995, Sol. Phys., 162, 101

Fröhlich, C., \& Lean, J. 2002, AN, 323, 203

Fröhlich, C., \& Wehrli, C. 2003, ftp://ftp.pmodwrc.ch/pub/publications/ SOH011-spm.pdf

Harvey, K. L. 1993, Magnetic Bipoles on The Sun, Univ. of Utrecht Hempelmann, A. 2002, A\&A, 388, 540

Hempelmann, A., \& Donahue, R. A. 1997, A\&A, 322, 835

Henry, G. W., Eaton, J. A., Hamer, J., \& Hall, D. S. 1995, ApJS, 97, 513

Horne, J. H., \& Baliunas, S. L. 1986, ApJ, 302, 757

Jenkins, J. M. 2002, ApJ, 575, 493

Jenkins, J. M., Doyle, L. R., \& Cullers, D. K. 1996, Icarus, 119, 244

Krivova, N. A., Solanki, S. K., Fligge, M., \& Unruh, Y. C. 2003, A\&A, 399, L1

Kurucz, R. 1991, in Stellar Atmospheres: Beyond Classical Models, ed. L. Crivellari, I. Hubeny, \& D. G. Hummer (Dordrecht: Kluwer Ac. Publ.), 441

Kurucz, R. 2000, http: //cfaku5 .harvard.edu

Lampton, M., Margon, B., \& Bowyer, S. 1976, ApJ, 208, 177

Lanza, A. F., Rodonò, M., Pagano, I., Barge, P., \& Llebaria, A. 2003, A\&A, 403, 1135 (Paper I)

Lawrence, J. K. 1987, J. Geophys. Res., 92, 813

Lawrence, J. K. 1988, Sol. Phys., 116, 17

Messina, S., Guinan, E. F., Lanza, A. F., \& Ambruster, C. 1999, A\&A, 347,249

Ortiz, A., Solanki, S. K., Domingo, V., Fligge, M., \& Sanahuja, B. 2002, A\&A, 388, 1036

Oster, L., Sofia, S., \& Schatten, K. 1982, ApJ, 256, 768

Pierce, K. 2000, in Allen's Astrophysical Quantities, the 4th edition, ed. A. N. Cox (Berlin: Springer-Verlag), Sect. 14.7

Priestley, M. B. 1981, Spectral Analysis and Time Series (London: Academic Press), Chap. 9

Press, W. H., Teukolsky, S. A., Vetterling, W. T., \& Flannery, B. F. 1992, Numerical Recipes in Fortran, the 2nd edition (Cambridge Univ. Press), Sect. 10.1

Radick, R. R., Lockwood, G. W., Skiff, B. A., \& Baliunas, S. L. 1998, ApJS, 118, 239

Rodonò, M., Cutispoto, G., Pazzani, V., et al. 1986, A\&A, 165, 135

Scargle, J. D. 1982, ApJ, 263, 835

Sofia, S., Oster, L., \& Schatten, K. 1982, Sol. Phys., 80, 87

Stellingwerf, R. F. 1978, ApJ, 224, 953

Strassmeier, K. G., Pichler, T., Weber, M., \& Granzer, T. 2003, A\&A, 411, 595

Unruh, Y. C., Solanki, S. K., \& Fligge, M. 1999, A\&A, 345, 635

Wadsworth, H. M. 1990, Handbook of Statistical Methods for Engineers and Scientists (New York: McGraw-Hill Publ. Co.) 